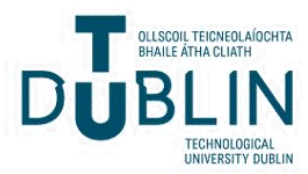

Technological University Dublin

ARROW@TU Dublin

2014-4

\section{Field Penetration in MRI-based Breast Models: a Numerical Investigation}

\author{
Giuseppe Ruvio \\ Technological University Dublin, Giuseppe.Ruvio@tudublin.ie \\ Raffaele Solimene \\ Seconda Università di Napoli \\ Antonio Cuccaro \\ Seconda Università di Napoli
}

See next page for additional authors

Follow this and additional works at: https://arrow.tudublin.ie/ahfrcart

Part of the Biomedical Engineering and Bioengineering Commons

\section{Recommended Citation}

G. Ruvio, R. Solimene, A. Cuccaro, and M. J. Ammann, "Field Penetration in MRI-based Breast Models: a Numerical Investigation", EuCAP 2014 - European Conference on Antennas and Propagation, The Hague, The Netherlands, pp. 316-319, 06/04/2014. doi:10.1109/EuCAP.2014.6901756

This Article is brought to you for free and open access by the Antenna \& High Frequency Research Centre at ARROW@TU Dublin. It has been accepted for inclusion in Articles by an authorized administrator of ARROW@TU Dublin. For more information, please contact arrow.admin@tudublin.ie, aisling.coyne@tudublin.ie, gerard.connolly@tudublin.ie.

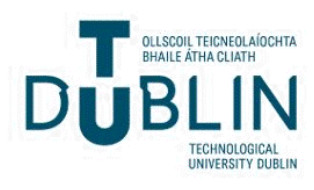


Authors

Giuseppe Ruvio, Raffaele Solimene, Antonio Cuccaro, and Max Ammann

This article is available at ARROW@TU Dublin: https://arrow.tudublin.ie/ahfrcart/57 


\title{
Field Penetration in MRI-based Breast Models: a Numerical Investigation
}

\author{
Giuseppe Ruvio $^{1,2}$, Raffaele Solimene ${ }^{2}$, Antonio Cuccaro ${ }^{2}$, Max J. Ammann ${ }^{3}$ \\ ${ }^{1}$ Trinity College University of Dublin, CTVR, Dublin, Ireland, ruviog@tcd.ie \\ ${ }^{2}$ Seconda Università di Napoli, Dipartimento di Ingegneria Industriale e dell'Informazione, DIII, Aversa, Italy \\ ${ }^{3}$ Dublin Institute of Technology, Antenna \& High Frequency Research Centre, AHFR, Dublin, Ireland
}

\begin{abstract}
The use of reliable computational tools is fundamental to investigate different aspects of microwave breast cancer imaging. From the development of high-definition and realistic numerical breast models, different coupling mechanisms and the reaction of different tissues to microwave signals can be characterized. In this paper, field penetration inside four numerical breast phantoms with varying adipose content is evaluated in the frequency range $0.5-10 \mathrm{GHz}$ across sagittal cuts.
\end{abstract}

Index Terms-Voxel models, Coupling, Breast cancer, Microwave imaging.

\section{INTRODUCTION}

As conventional X-ray mammography was shown to be limited due to poor benign/malignant tissue contrast [1], recent interest in active microwave based diagnostic approaches has proliferated. If higher spatial resolution is achievable by using $\mathrm{X}$-ray ionizing radiation, radio frequency technology offers a suitable dielectric contrast between normal and diseased breast tissues which can be in the order of $2: 1$. RF breast cancer imaging technology can therefore have consequent high social and cost impact. These important potential outcomes have triggered the investigation of many microwave imaging techniques, aimed at detecting, localizing and identifying tumors in breast tissues [2, 3].

Microwave imaging research has also significantly developed towards sophisticated numerical and experimental breast models for the evaluations of antennas, radar configurations and algorithms. This aspect is particularly important for a research area which explores non-invasive cancer diagnostics where clinical trials represent the ultimate experimental assessment for a novel imaging system. In [4], [5] ex vivo breast tissues were electrically characterized across a large part of the microwave spectrum and described in terms of accurate Cole-Cole dispersion model. Alongside the electrical characterization of tissues, dedicated in-house software has been developed by several research groups and many of them have reached a very accurate electrical model of both benign and malignant tissues. At the same time, the strong interest in biomedical imaging applications in the range of radio frequencies has triggered the integration of specific tools into commercial code platforms. Reliable and complex numerical simulations enable a deep understanding of non- apparent coupling and field propagation mechanisms. This assumes a particular importance for breast cancer detection as its performance strictly relates to the power delivered inside deep tissue layers. Breast tissues react to RF signals in different ways but three major phenomena can be generally observed:

- $\quad$ Skin largely screens RF energy;

- $\quad$ Adipose parts couple better;

- Tissues with high water-content (i.e. glandular) strongly scatter.

These mechanisms occur in very heterogeneous scenarios that change from patient to patient. However, according to their radiographic density, defined by the American College of Radiology [6], four categories were defined as follows: almost entirely fat $(<25 \%$ glandular tissue), scattered fibroglandular tissue $(25-50 \%$ glandular), heterogeneously dense breast (51$75 \%$ glandular), and very dense breast ( $>75 \%$ glandular).

While showing simulation capabilities of CST Microwave Studio for breast cancer detection, this numerical evaluation explores the penetration of RF signals through breast tissues in realistic heterogeneous scenarios. Results provide valuable information regarding expected power levels inside different categories of breast with varying fibroglandular / adipose ratios and for different frequencies.

\section{NUMERICAL INVESTIGATION}

In this paper, four 3-D numerical breast phantoms are selected within the Wisconsin's repository [7] to represent the four categories above described. The chosen models are labeled as "Mostly Fatty ID071904", "Scattered Fibroglandular ID012204", "Heterogeneous Dense ID080304" and "Very Dense ID012304", respectively. The phantoms are derived from T1-weighted magnetic resonance images (MRIs) of patients in a prone position. Each phantom comprises a 3-D grid of cubic voxels of $1 \mathrm{~mm} \times 1 \mathrm{~mm} \times 1 \mathrm{~mm}$ and is imported into CST Microwave Studio [8]. The structural heterogeneity of the breast incorporates the Cole-Cole dispersive dielectric models of ten different tissues reported by Lazebnik in [4], [5] and the CST 4-Cole-Cole dispersion models (Table I). The breast voxel models are immersed in coupling medium with $\varepsilon_{\mathrm{r}}$ $=10$. For simplicity and generality, no dispersion is assumed for the coupling medium. Ideal probes are inserted inside the 


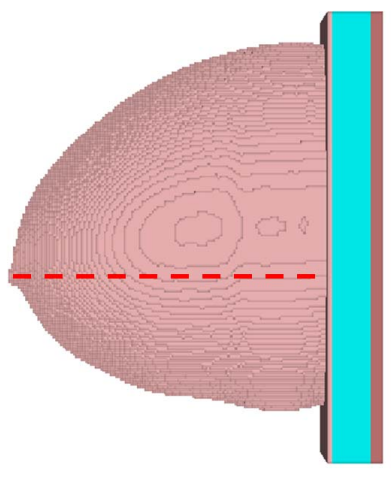

Mostly Fatty

ID071904

(a)

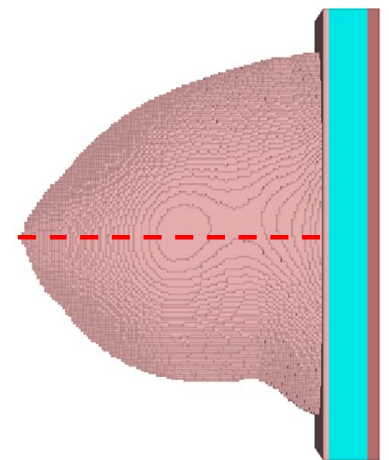

Scattered Fibroglandular ID012204

(b)

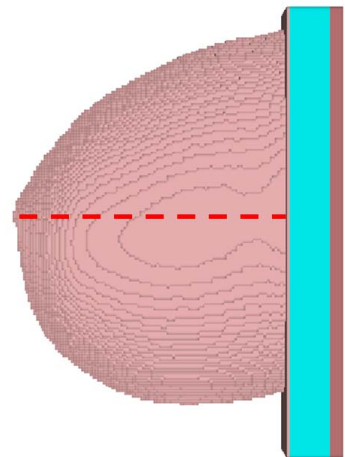

Heterogeneous Dense ID080304

(c)

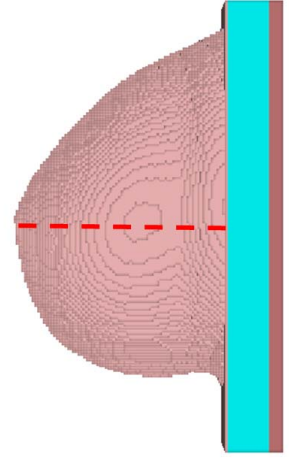

Very Dense ID012304

(d)

Figure 1: Voxel breast models and reference cuts.

Table I

Single-pole and 4-pole Cole-Cole parameters for the dielectric properties associated with voxel breast models

\begin{tabular}{|c|c|c|c|c|c|}
\hline & $\varepsilon_{\infty}$ & $\Delta \varepsilon$ & $\tau[\mathrm{ps}]$ & $\alpha$ & $\sigma_{\mathrm{s}}[\mathbf{S} / \mathbf{m}]$ \\
\hline Fatty-1 & 2.293 & 0.141 & 16.40 & 0.251 & 0.002 \\
\hline Fatty-2 & 2.908 & 1.200 & 16.88 & 0.069 & 0.020 \\
\hline Fatty-3 & 3.140 & 1.708 & 14.65 & 0.061 & 0.036 \\
\hline Transitional & 4.031 & 3.654 & 14.12 & 0.055 & 0.083 \\
\hline Fibroconnective/glandular-1 & 9.941 & 26.60 & 10.90 & 0.003 & 0.462 \\
\hline Fibroconnective/glandular-2 & 7.821 & 41.48 & 10.66 & 0.047 & 0.713 \\
\hline Fibroconnective/glandular-3 & 6.151 & 48.26 & 10.26 & 0.049 & 0.809 \\
\hline
\end{tabular}

\begin{tabular}{|c|c|c|c|c|c|c|c|c|c|c|c|c|c|c|}
\hline & $\boldsymbol{\varepsilon}_{\infty}$ & $\Delta \varepsilon 1$ & $\tau 1$ [ps] & $\alpha 1$ & $\Delta \varepsilon 2$ & $\tau 2[\mathrm{~ns}]$ & $\alpha 2$ & $\Delta \varepsilon 3$ & $\tau 3$ [ps] & $\alpha 3$ & $\Delta \varepsilon 4$ & $\tau 4$ [ps] & $\alpha 4$ & $\begin{array}{c}\sigma_{\mathrm{s}} \\
{[\mathrm{S} / \mathrm{m}]}\end{array}$ \\
\hline Muscle & 4 & 50 & 7.234 & 0.100 & 7000 & 353.678 & 0.1 & $1.20 \mathrm{E}+6$ & 318.310 & 0.1 & $2.5 \mathrm{E}+7$ & 2.274 & 0 & 0.2 \\
\hline Skin & 4 & 39 & 7.958 & 0.100 & 280 & 79.577 & 0 & $3.0 \mathrm{E}+4$ & 1.592 & 0.160 & $3.0 \mathrm{E}+4$ & 1.592 & 0.2 & 0 \\
\hline
\end{tabular}

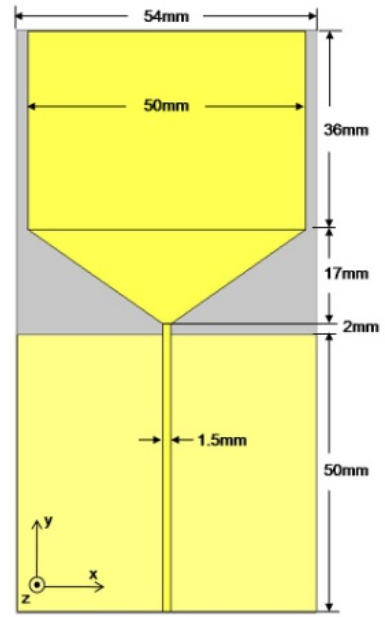

Figure 2: Geometry of the coplanar monopole. different depth levels in the frequency range from 0.5 to 10 $\mathrm{GHz}$. In the aim of crossing different layer distributions, one of ideal probes are placed along the axes in the sagittal planes corresponding to the nipple area so to include all types of breast tissues (Fig. 1).

This evaluation is initially carried out with the breast irradiated by an ideal plane wave in order to focus just on the field penetration and disregard coupling effects between source and breast. Then, the field intensity is calculated when a coplanar monopole is considered as a source in close proximity to the breast (i.e. $\lambda_{\mathrm{m}} / 2$ at $5 \mathrm{GHz}$ which is equal to $9.48 \mathrm{~mm}$ in the coupling medium). The geometry of the co-planar monopole is shown in Fig. 2. The dielectric substrate used is 1.58-mm thick Taconic CER10 material. The bottom edge of the radiating element was beveled to extend a good impedance match over a larger frequency range. 

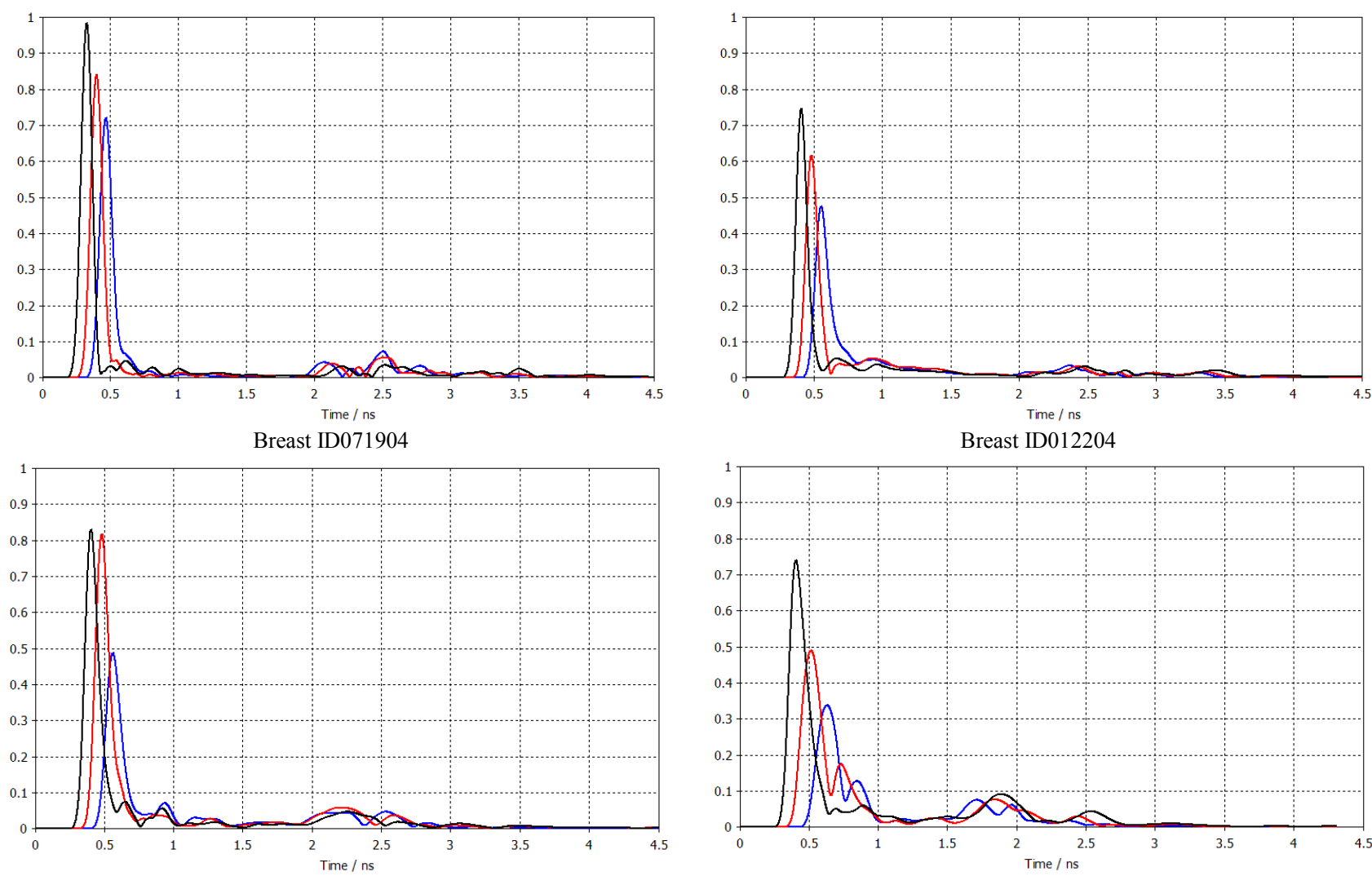

Breast ID080304

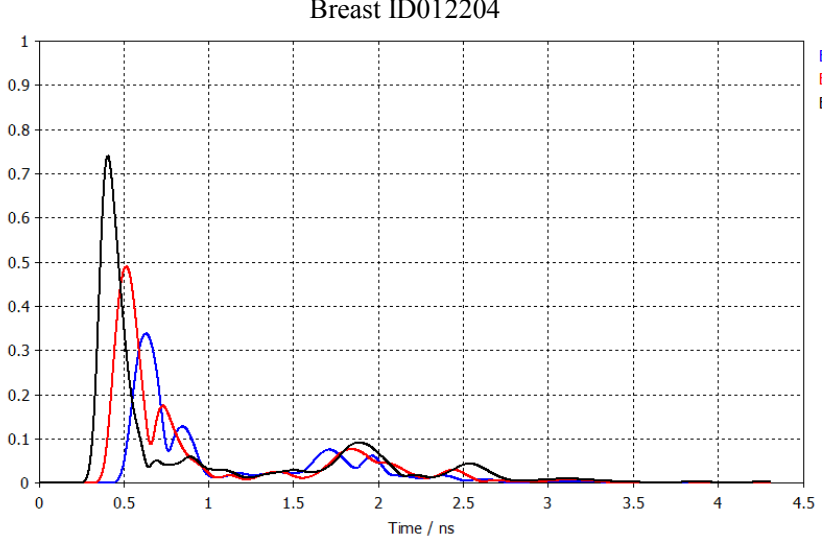

Breast ID012304

Figure 3: Time signals at Probes 1, 2 and 3 with ideal plane wave radiation (Probe 1 - black trace, Probe 2 - red trace, Probe 3 - blue trace).

Three probes were considered to monitor the field penetration in the time domain, with Probe 1, 2 and 3 placed at $0.5,1.5$ and $2.5 \mathrm{~cm}$ from the skin layer, respectively.

\section{RESULTS AND DISCUSSION}

Figures 3 show the signals captured at Probe 1, 2 and 3 for the four breast typologies investigated with an ideal plane wave used as source. All traces were normalized and displayed on the same time interval $0-7$ ns. The stimulation is performed with a Gaussian pulse defined in the frequency range $0-10 \mathrm{GHz}$. It can be observed that, as expected, the signal undergoes stronger attenuation in breast models with larger fibroconnective/glandular tissue distribution. Another expected result is the increasing time-delay between probes in denser breast models. This aspect verifies the longer time needed by the signal to travel across highest-water-content materials. When an ideal plane is adopted as radiator, coupling issues between source and breast do not occur and the shape of the Gaussian pulse does not appear dramatically distorted. However, ringing effects can be noticed especially for highly heterogeneous and dense models.

The effects of coupling occurring between realistic antennas and breast can be appreciated in Fig. 4 where the coplanar monopole in Fig. 2 was adopted as source. The effects observed with the ideal source such as increasing attenuation and time-delay towards deeper probes in denser breasts are still present. But they add up with pulse-distortion degradation due to the antenna and the complex reactive fields that appear between the antenna and the breast. Such distortion is very hard to characterize a priori as it strongly depends on the distance between, the antenna and the breast, the shape and the tissue distribution of the breast.

Table II

Peak-value variation between Probe 1 and 3

Ideal plane wave

\begin{tabular}{cc}
\hline \hline Breast & Field variation [\%] \\
\hline ID071904 & 27 \\
ID012204 & 36 \\
ID080304 & 40 \\
ID012304 & 54 \\
\multicolumn{2}{c}{ Coplanar monopole } \\
\hline \hline Breast & Field variation [\%] \\
ID071904 & 68 \\
ID012204 & 80 \\
ID080304 & 84 \\
ID012304
\end{tabular}


Although arbitrary choices such as the breast models selected, the location of the probes and the coplanar monopole geometry relative were made in this article, relative comparisons are informative. The field intensity variation between Probe 1 and $3(0.5$ and $1.5 \mathrm{~cm}$ from the skin layer, respectively) for different breast models are under investigation was calculated and summarized in Table II. Relative variations show a challenging attenuation of the field when it travels $1.5 \mathrm{~cm}$ inside the breast.

This numerical investigation shows the suitability of CST Microwave Studio for the analysis of electromagnetic phenomena in presence of highly accurate MRI-based voxel models. Moreover, the difference between plane wave and monopole on the signal degradation justifies the employment of ideal sources only for preliminary evaluation of breast imaging algorithms. On the other hand, the strong dependence of the signal distortion on coupling effects motivates the interest on algorithms which operate with limited a priori antenna response information [9].

\section{AKNOWLEDGEMENT}

This work was supported by Italian Ministry of University and Research through the FIRB initiative under the project MICENEA (RBFR12A7CD), POR Campania FSE 2007/2013-"MASTRI" and Science Foundation Ireland.

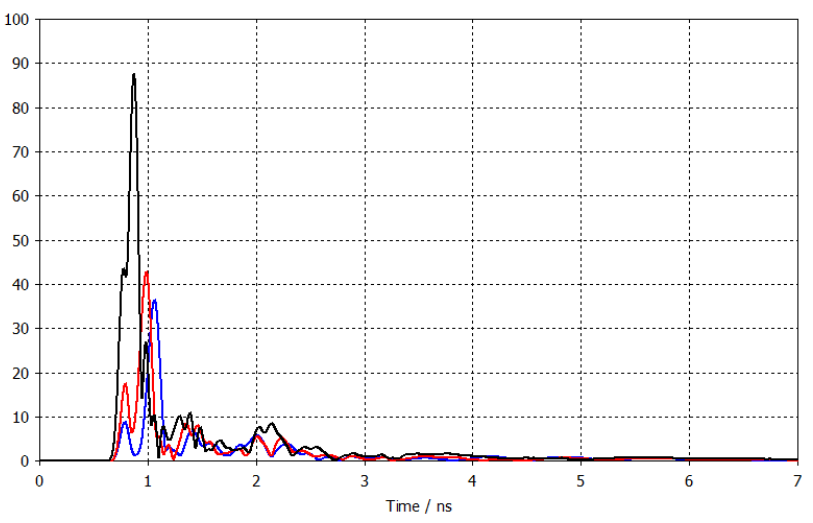

Breast ID071904

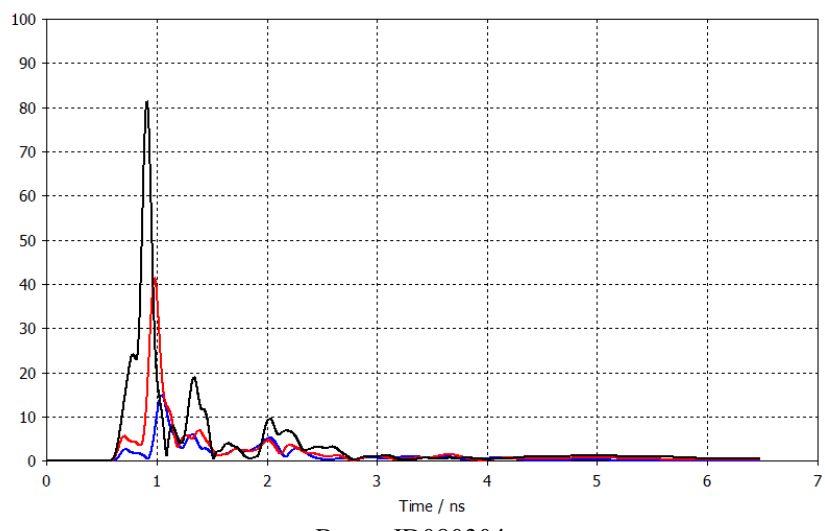

Breast ID080304

\section{REFERENCES}

[1] [1] J.G. Elmore, M.B. Barton, V.M. Moceri, S. Polk, P.J. Arena, and S.W. Fletcher, "Ten-year risk of flase positive screening mammograms and clinical breast examinations," New Eng. J. Med., vol. 338, no. 16, pp. 1089-1096, 1998.

[2] E.C. Fear, S.C. Hagness, P.M. Meaney, M. Okoniewski, and M.A. Stuchly, "Enhancing breast tumor detection with near-field imaging," IEEE Microwave Magazine, vol. 3, no. 1, pp. 48-56, Aug 2002.

[3] N.K. Nikolova, "Microwave Imaging for Breast Cancer," IEEE Microwave Magazine, vol. 12, no. 7, pp. 78-94, Dec 2011.

[4] M. Lazebnik, L. McCartney, D. Popovic, C. B. Watkins, M. J. Lindstrom, J. Harter, S. Sewall, A. Magliocco, J. H. Booske, M. Okoniewski, and S. C. Hagness, "A large-scale study of the ultrawideband microwave dielectric properties of normal breast tissue obtained from reduction surgeries," Physics in Medicine and Biology, vol. 52, pp. 2637-2656, April 2007.

[5] M. Lazebnik, D. Popovic, L. McCartney, C. B. Watkins, M. J. Lindstrom, J. Harter, S. Sewall, T. Ogilvie, A. Magliocco, T. M. Breslin, W. Temple, D. Mew, J. H. Booske, M. Okoniewski, and S. C. Hagness, "A large-scale study of the ultrawideband microwave dielectric properties of normal, benign, and malignant breast tissues obtained from cancer surgeries," Physics in Medicine and Biology, vol. 52, pp. 6093-6115, 2007.

[6] American College of Radiology, Breast Imaging Reporting and Data System (BI-RADS®), $4^{\text {th }}$ ed., 2003.

[7] E. Zastrow, S. K. Davis, M. Lazebnik, F. Kelcz, B. D. Van Veen, and S. C. Hagness, "Database of 3D Grid-Based Numerical Breast Phantoms for use in Computational Electromagnetics Simulations," online http://uwcem.ece.wisc.edu/MRIdatabase/InstructionManual.pdf.

[8] Online http://www.cst.com.

[9] G. Ruvio, R. Solimene, A. Cuccaro, and M. J. Ammann, "Comparison of Non-Coherent Linear Detection Algorithms Applied to a 2-D Numerical Breast Model", IEEE Antennas and Wireless Propagation Letters, vol. 12, pp. 853 - 856, 2013.

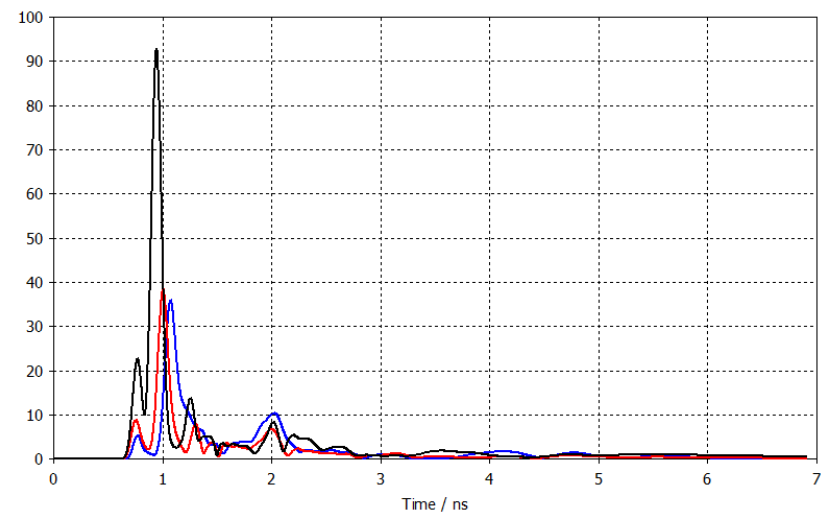

Breast ID012204

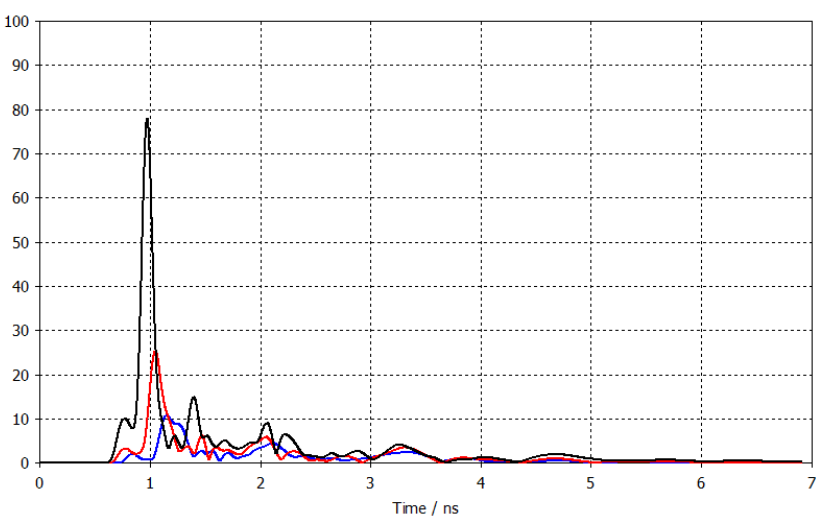

Breast ID012304

Figure 4: Time signals at Probes 1, 2 and 3 with coplanar monopole (Probe 1 - black trace, Probe 2 - red trace, Probe 3 - blue trace). 